\title{
Assembly and disassembly of mammalian Chromosome pellicle
}

NI ZumeI* ,JE LitTle**ANd LuJi SHI(LC SZE)*

* Shanghai Institute of Cell Biology, Acadmia Sinica, Shanghai 200031, China.

** Department of Cell Biology, Faculty of Science and Engineering, University of Ottawa, Ottawa. Ontario, Canada. (KIN)

\begin{abstract}
By means of indirect double immunofluorescent staining, the coordination of PI antigen and perichromonucleolin (PCN), the constituent of nuclear periphery and nucleolus respectively, in the assembly and disassembly of chromosome pellicle during mitosis was studied. It was found that in 3T3 cells, during mitosis PI antigen began to coat the condensing chromosome surface earlier than PCN did. However, both of them completed their coating on chromosome at approximately the same stage of mitosis, prometaphase metaphase. The dissociation of PI antigen from chromosome pellicle to participate the formation of nuclear periphery took place also ahead of that of PCN. At early telophase PI antigen had been extensively involved in the formation of nuclear periphery, while PCN remained in association with the surface of decondensing chromosomes. At late telophase, when PI antigen was localized in an fairly well formed nuclear periphery, PCN was in a stage of forming prenucleolar bodies.
\end{abstract}

Key words: chromosome pellicle, assembly, disassembly, PI antigen, perichromonucleolin (PCN), indirect double immunofluorescent staining, mitosis.

\section{INTRODUCTION}

The existence of the pellicle or coat of metaphase and anaphase chromsomes was once questioned by many cell biologists. Recently, from the immunofluorescence studies on cells, evidence has been found that the pellicle is a real structure of the condensed chromosomes (metaphase and anaphase chromosomes) [1, 2, 3, 4]. Some of its chemical constituents have been characterized by immuno-blotting. Their distribution and changes during interphase and mitosis have been investigeted. They are derived from 
different nuclear structures (nuclear periphery and nucleoli)and take part in the assembly and disassembly of chromosome pellicle during prophase, and during telophase and interphase respectively. The sequential cytological events of each of these pellicle constituents are now known fairly well. However, more precise coordination of these events is still lacking. Double immunofluorescent staining enables the study of the distribution of 2 antigens in the same cell. By means of this technique and making use of the pellicle antibodies, namely, PI and PCN specific antibody at hand, we have recently looked into this problem. The results of our studies on 3T3 cells are presented.

\section{MATERIALS AND METHODS}

\section{Cell culture}

Mouse 3T3 cell were cultured over coverslips with 5\% $\mathrm{CO}_{2}$ in Dulbecco's modified Eagle's medium supplemented with $10 \%$ fetal calf serum, $100 \mu / \mathrm{ml}$ penicillin, $100 \mu \mathrm{g} / \mathrm{ml}$ streptomycin and $0.25 \mu \mathrm{g} / \mathrm{ml}$ fungizone.

\section{Antibodies}

Four antibodies were used in the present studies. Two of them were primary, namely PI antibody and PCN specific antibody. The former is a mouse monoclonal antibody which has been described by Chaly et al [3] and the latter was screened from scleroderma patients as described by Shi et al [9]. The secondary antibodies used were rabbit anti mouse IgG preabsorbed with human IgG, conjugated with tetra methyl rhodamine isocyanate and rabbit anti human IgG, conjugated with fluorescein isocyanate.

\section{Fixation}

Double fixation was adopted. The coverslips with cells, after short rinse in PBS, were fixed with the first fixative (3\% paraformaldehyde in PBS, $\mathrm{pH}$ 7.0) for 30 sec, followed by treatment with $1 \%$ Triton $\mathrm{X} 100$ in stabilization buffer (SB) $\left(25 \mathrm{~m} M\right.$ imidazol, $25 \mathrm{~m} M$ KCI, $0.25 \mathrm{mM} \mathrm{MgCl} 2 \cdot 6 \mathrm{H}_{2} \mathrm{O}, 0.5 \mathrm{~m} M$ EGTA, 0. 05m $M$ EDTA, 0. $5 \mathrm{~m} M 2$ mercaptoethanol, $2 M$ glycerol $\mathrm{pH}$ 6. 5) for $60 \mathrm{~min}$, washing twice in SB for $30 \mathrm{sec}$ each. Then the cells were fixed with the second fixative ( $0.2 \%$ glutaraldehyde in SB) for 4 min, followed by treatment with sodium borohydride solution ( $\mathrm{lmg} / \mathrm{ml}$ in PBS pH 7.0), 3 times, 4 min each, and washing in PBS, 3 times, 5 min each.

\section{Indirect single immunofluorescent staining}

The fixed and washed cells were incubated with primary antibody for $45 \mathrm{~min}$ at room temperature and washed with PBS 3 times, 4 min each. They were then incubated with secondary antibody for $45 \mathrm{~min}$ at room temperature. After they had been washed with PBS 3 times, 4 min each, they were counter stained with Hoechst 33258 for 2 min and mounted in glycerol PBS phenylene diamine.

\section{Indirect double immunoflourescent staining}

The fixed and washed cells were first successively incubated with PI antibody and rabbit anti mouse IgG preabsorbed with human IgG and rhodamine conjugated as described in the single immunofluorescent staining. After being washed 3 times in PBS, 4 min each, once in 0.15\% gelatin in PBS, 20 min, and 3 times in PBS, 10 min each, the cell were incubated in successing with PCN specific antiserum and rabbit anti human IgG FITC conjugated. They were then washed 3 times in PBS, 4 min each, counterstained with Hoechst 33258 in PBS for 2 min and mounted.

\section{RESULTS}

\section{1) The effect of double fixation adopted on the distribution and changes of the pellicle}




\section{antigens.}

Since 3T3 cells were fixed with double fixation method which is different from those used in our previous studies, the distribution and changes of both PI antigen and PCN were checked. In general, the observations on the double-fixed cell confirmed our previous results [3, 9]. However,in the case of PI antigen some minor differences were noted. In double-fixed cells in addition to nuclear periphery, the cytoplasm of interphase cells was distinctly stained by PI. From metaphase to telophase, some cytoplasmic particles and the distal end of each half of stem body were stained by PI too (see Fig 1, Row $8 \mathrm{c}$ ).

\section{2) Preliminary observations on indirect double immunofluorescent staining.}

Technically, direct double immunofluorescent staining is more specific in comparison with indirect method. However, the method is less sensitive and requires the preparation of labeled primary antibodies. Thus, indirect double immunofluorescent staining was adopted. Since the quality of the secondary antibodies is important in this method, some preliminary observations were made. When both secondary antibodies were used without pretreatment, PCN and PI antigen were distinctly cross - stained (data not shown). When the rabbit anti-mouse IgG was preabsorbed with human IgG, the results were dependent upon the sequential order of the staining. There was no distinct cross-staining of PCN and PI antigen when the order is: PI antibody and anti -mouse IgG (pre -absorbed with human IgG) staining, followed by PCN-specific antibody and anti -human IgG. This order was here used. We had also tried the mixed primary and secondary antibodies, but not successful.

\section{3) The coordination of PI antigen and PCN in the assembly and disassembly of the chro- mosome pellicle.}

From the preliminary observations the indirect immunoflourescent staining as described above was used. Our results are shown in Fig 1 Here, each row consists of three photomicro - graphs taken from the same cell at a particular stage of the cell cycle. One of them indicates the stage of the cell cycle at which the cell reached shown by Hoechst 33258 staining, and the other 2 display respectively, the state of distribution of PCN and PI antigen in the same cell. The set of the rows shows successive stages of mitosis. At interphase, PI antigen was localized in nuclear periphery, while PCN was compartmentalized in the nucleoli (Fig 1 Row a,b,c). In early prophase, the coating of PI antigen onto the surface of prophase chromosomes had already proceeded to a notable extent when PCN lumps just started to disperse into irregular masses (Fig 1 Row 2 a,b,c). When mitosis had progressed to late prophase, the coating of PI antigen onto chromosomes became extensive and that of PCN was carried out only to a prominent degree (Fig 1 Row3 a, b ,c - 4 a,b,c). However, the coatings of both PI antigen and PCN were completed approximately at the prometaphase - metaphsase (Fig 1 Row 5 a,b ,c-6 a ,b, c). At early telophase, when PCN still delineated individual chromosomes, PI antigen had already been dissociated from most part of the chromosomes to participate in the formation of nuclear periphery, (Fig 1 Row 7 a,b,c). By the time when PI antigen had been localized in the fairly well formed periphery, PCN was in a stage of forming 
Assembly and disassembly of mammalian chromosome pellicle

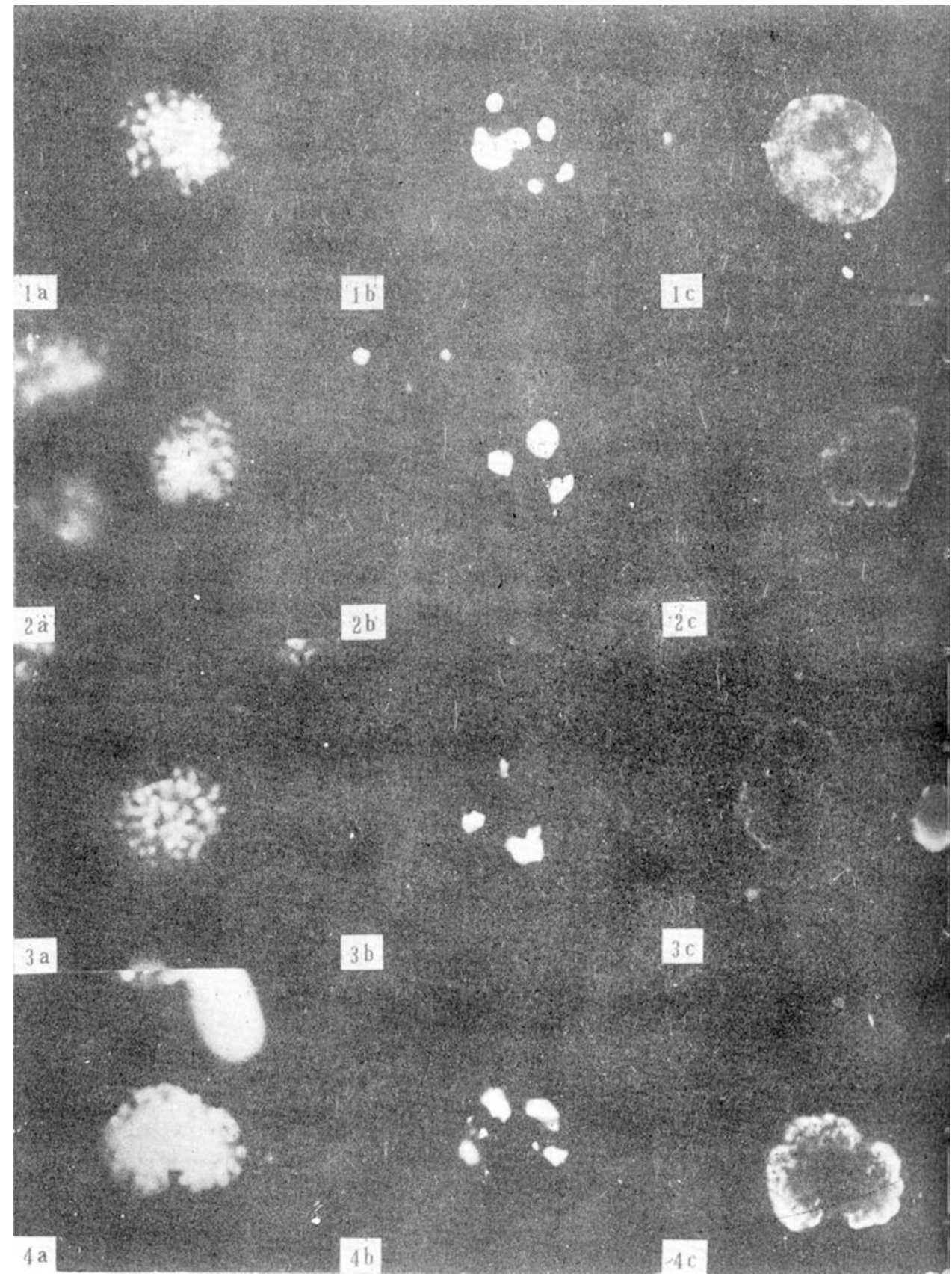

Fig 1. Row 1 interphase; Row 2-5 successive stage of prophase; Row 6 metaphase; Row 7 early with Hoechest 33258, b stained with PCN antibody, c stained with PI antibody. 
Ni ZM et al.

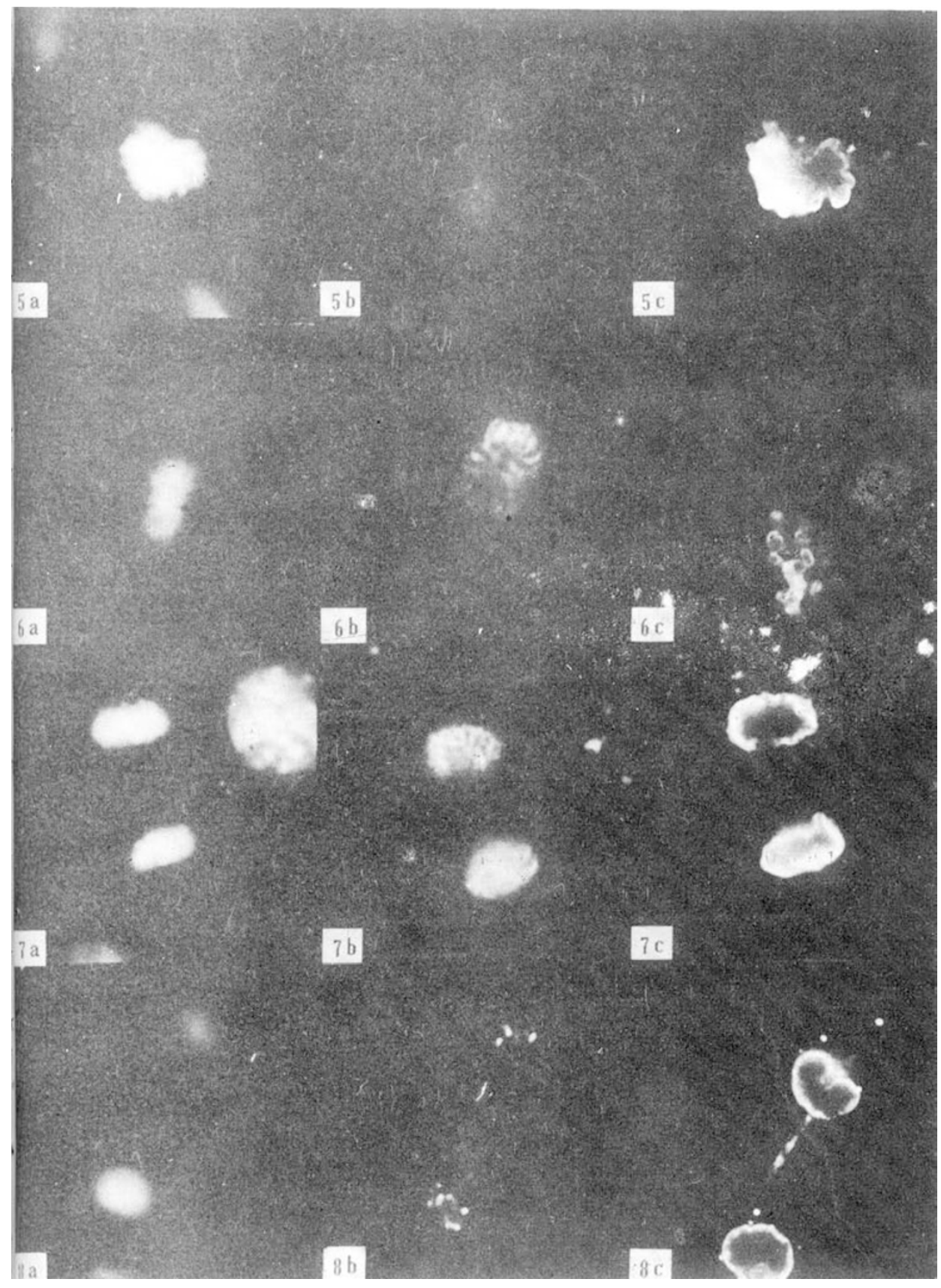

telophase; Row 8 late telophase. In each row a, b, c come from the same cell.a counterstained 
Assembly and disassembly of mammalian chromosome pellicle

prenucleolar bodies (Fig 1 Row 8 a. b. c). From these observations, we concluded that (1) in the assembly of Chromosome pellicle, PI antigen starts to coat the surface of condensing chromosomes earlier than PCN and they finish the coating process at approximately the same time (prometaphase metaphase), and (2) in the disassembly of chromosome pellicle, the dissociation of PI antigen from the decondensing chromosomes also precedes that of PCN.

\section{DISCUSSION}

Our findings showed that chromosome pellicle is not assembled and disassembled simultaneously by its constituents from different structural origins. The coating of PI antigen to the surface of condensed chromosomes starts earlier than that of PCN, but both coatings finish at approximately the same time. Likewise, the dissociation of PI antigen from the surface of decondensing chromosomes goes also ahead of that of PCN. These may mean that the disassembly of nuclear periphery is proceeding slower than that of the nucleoli, while the assembly of former is carried faster than that of the latter, PI antigen, M108 antigen and perichromin are all constituents of the nuclear periphery. Whether these pellicle constituents are derived from the same structure or are concomitantly incorporated into and disassembled from chromosome pellicle remains to be investigated.

The present results and those by others suggested the existence of an assembly disassembly cycle of the surface of condensed chromosomes (metaphase and anaphase chromosomes) during mitosis. This surface has a high affinity to the PI antigen, M108 antigen, perichromin and PCN. It is progressively assembled during prophase to its completion at metaphase and is gradually disassembled during telophase up to its dissolution at interphase. It might be corresponding to the epichromatin of Howell and Hsu [5]. Its assembly disassembly cycle may be a result of chromosome condensation decondensation cycle, since the latter is an independent process during mitosis [6]. The changes in distribution Of M 108 during mitosis as shown by the PCN study suggested that the surface of condensed chromosomes starts to form at $\mathrm{G}_{2}$ of the cell cycle [4]. Up to the present, some building material of chromosome pellicle are known to come from nuclear periphery (PI antigen, M108 antigen, perichromin) and nucleoli (PCN). The assembly disassembly cycles of these nuclear structures are well documented [1,2,3, $7,8]$, Thus in relation to the assembly disassembly of chromosome pellicle, there are 3 such cycles of nuclear structures, namely nuclear periphery, nucleolus and the surface of condensed chromosomes. Therefore, during mitosis, there are all together 4 interrelated cycles, Previous and the present studies showed that the assembly disassembly cycle of chromosome pellicle is approximately in phase with that of the surface of condensed chromosomes and in antiphase with those of nuclear periphery and nucleoli.

The phase relationship among the 4 assembly disassembly cycles mentioned suggested a possible origin of the assembly disassembly cycle of chromosome pellicle. Recent findings showed that when the assembly of nuclear periphery is inhibited by depletion of lamins, the morphological structure of metaphase chromosomes is well maintained and the formation of nucleoli and the reconstruction of nucleus are inhibited [9, 
10]. These suggested that the assembly of one structure is important to the disassembly of the other and vise versa. The disassembly of nuclear periphery and nucleoli during prophase progressivly set free their constituents, some of these constituents such as PI antigen, M 108 antigen, perichromin from nuclear periphery and PCN from nucleoli are bound by the forming surface of condensed chromosomes due to their affinity to it, resulting progressive assembly of chromosome pellicle. As the new nuclear periphery and nucleoli are assembling during telophase, the disassembly of the surface of condensed chromosomes releases gradually the pellicle constituents for their reconstruction. Thus the assembly- disassembly cycle of chromosome pellicle could be considered as a result of the interaction between the surface of condensed chromosomes and those of the pellicle-related nuclear structures. However, other possibilities cannot be ruled out at present. Further experimental studies are needed to clarify the problem.

\section{REFERENCES}

[1] Chaly N, Bladon T, Setterfeild G, Little JE, Kaplan JG, Brown DL. Changes in distribution of nuclear matrix antigens during mitotic cycle. J Cell Biol 1984; 99:661-71.

[2] Mckeon FD, Tuffanelli DC, Kobayashi S,Kerschner MW. The redistribution of a conserved nuclear envelope protein during the cell cycle suggests a pathway for chromosome condensation. Cell 1984; 36:83-92.

[3] Shi LC (Sze LC), Ni ZM, Zhao S, Wang G, Yang Y. The involvement of a nucleolar component, perichromonucleolin, in chromosome condensation. Proc Natl Acad Sci USA 1987; 84: 7953-7956.

[4] Wataya-Kaneda, Kaneda Y, Sakurai T, Sugawa H, Uchida T. A monoclonal antibody against the nucleus reveals the presence of common protein in the nuclear envelope, the perichromosomal region and cytoplasmic vesicles. J Cell Biol 1987; 104: 1-7

[ 5 ] Hawell WM, Hsu TC. Chromosome core structure revealed by silver staining. Chromosoma 1979; 73: 61-6.

[6] Newport J, Spann T, Wilsin K, Dunphy W, Kanki J. Regulation of nuclear assembly and breakdown in vitro. J Cell Biol 1986; 103:47a.

[ 7 ] Jordan EG, Cultis CA (editors). The nucleolus (Society for experimental biology 1982 Seminar Series 15) The Press Syndicate of the University of Cambridge, Cambridge, UK

[ 8 ] Mckeon FD, Tuffanelli DC, Fukuyama DL, Kerschner MW. Autoimmune response against conserved determinants of nuclear envelope proteins in patient with linear scleroderma. Proc Natl Acad Sci USA 1983; 80:4347 -78 .

[ 9 ] Benavente R, Krobe G. Involvement of nuclear lamins in post mitotic reorganization of chromatin as demonstrated by micro - injection of lamins antibodies. J Cell Biol 1986; $103: 1847$ - 54.

[10 ] Burke B, Gerace L. A cell free system to study reassembly of the nuclear envelope at the end of mitosis. Cell $1986 ; 44: 639-52$.

Received 20 - 12 - 1991. Accepted 30 - 1 - 1992. 